\title{
Effects of accelerated crucible rotation on segregation and interface morphology for vertical Bridgman crystal growth: Visualization and simulation
}

\author{
Y.C. Liu ${ }^{\mathrm{a}}$, B. Roux ${ }^{\mathrm{b}}$, C.W. Lan ${ }^{\mathrm{a}, *}$ \\ ${ }^{a}$ Department of Chemical Engineering, National Taiwan University, Taipei 10617, Taiwan, ROC \\ ${ }^{\mathrm{b}}$ Laboratoire Modélisation et simulation numérique en mécanique, L3M, CNRS-Universités d'Aix-Marseille, France \\ Received 22 April 2006; received in revised form 8 January 2007; accepted 29 January 2007 \\ Communicated by A.G. Ostrogorsky \\ Available online 12 March 2007
}

\begin{abstract}
The effects of accelerated crucible rotation technique (ACRT) on the segregation, interface shape, and morphological instability during vertical Bridgman (VB) crystal growth of succinonitrile (SCN) containing $0.064 \mathrm{wt} \%$ acetone were investigated by visualization experiments and computer simulation. It was found that ACRT was effective in reducing time-averaged constitutional supercooling and thus the morphological instability. However, at low rotation amplitudes, the morphology was less stable and the breakdown area was found to be wider as a result of flow transition. The simulated interface shapes were in good agreement with the observed ones. In addition, the onset of morphological breakdown was consistent with the numerical prediction based on the time-averaged constitutional supercooling.
\end{abstract}

(C) 2007 Elsevier B.V. All rights reserved.

PACS: 44.25. +f; 47.27.Te; 81.10.Fq; 02.60.c6; 02.70.Fj

Keywords: A1. Accelerated crucible rotation; A1. Convection; A1. Interface morphology; A2. Bridgman method

\section{Introduction}

The control of segregation and interface morphology is important in the vertical Bridgman (VB) process, and the amelioration of buoyancy-driven convection is crucial. Because of a lack of control over the stirring condition, the introduction of forced convection by external forces for obtaining a favorable growth condition is often necessary. The accelerated crucible rotation technique (ACRT) proposed by Scheel and Schulz-DuBois [1] has been a useful means because of its simplicity and versatility [2,3]. By controlling the acceleration cycle patterns and the interactions with the buoyancy-driven convection, one could control the melt mixing at either an enhanced mixing or diffusion-limited mode [3-6]. The control also depends

\footnotetext{
${ }^{*}$ Corresponding author. Tel./fax: + 886223633917.

E-mail address: cwlan@ntu.edu.tw (C.W. Lan).
}

on the ratio of the melt depth to the size of Ekman cells, the buoyancy-driven convection, and the Taylor vortices due to the Taylor-Görtler instability near the ampoule wall [7,8]. A few numerical studies have been made to better understand the interplay of the flow and segregation, e.g. [7-11]. Numerical benchmark comparison can be found as well [11]. However, the effect of ACRT on the interface shape and morphological instability during Bridgman crystal growth still has not yet been well addressed, particularly on the morphological instability.

The morphological instability, as a result of constitutional supercooling, is frequently encountered in the growth of an alloy crystal. Therefore, to keep a stable planar growth interface, a low solidification speed is necessary in practice. During crystal growth, due to the solute segregation at the interface, the solute boundary layer develops, and thus the solidification temperature changes, in front of the growing interface. Once the melt 
temperature is lower than the solidification temperature, constitutional supercooling occurs. The constitutional cooling at the interface could be examined easily by the superheating gradient $\Delta G$ [12] defined by

$\Delta G=G-m \frac{\mathrm{d} C}{\mathrm{~d} n}$,

where $G$ is the temperature gradient, $m$ the slope of the liquidus line in the phase diagram, and $\mathrm{d} C / \mathrm{d} n$ is the normal solutal gradient. When $\Delta G$ is less than zero, constitutional supercooling occurs. As the supercooling is built up to a certain degree, the interfacial energy is no longer able to keep a planar growth front. The planar interface is broken down leading to a cellular or dendritic structure, which is detrimental to crystal quality. Since both the thermal and solutal gradients in Eq. (1) are greatly affected by convection, ACRT has been shown effective in the control of the morphological stability as well $[2,3]$. To investigate the effect of ACRT on the morphological instability, a transparent growth system should be useful in that respect. Moreover, as the visualized results are compared with numerical simulation, the role of ACRT can be understood better and the technique can be used more effectively.

In this paper, we present the experimental results from a transparent Bridgman growth system, where succinonitrile $(\mathrm{SCN})$ containing $0.064 \mathrm{wt} \%$ acetone was used as the model material. Computer simulations are also carried out to interpret the effects of ACRT through the calculated heat flow and solute fields. In the next section, the experimental system and the numerical method are described briefly. Section 3 is devoted to results and discussion, and is followed by a short conclusion.

\section{Experiments and numerical simulation}

SCN containing $0.064 \mathrm{wt} \%$ acetone was directionally solidified in a transparent VB system that was installed at the center of a rotating table, as shown in Fig. 1a. Before experiments, SCN (Acros Inc., about 99\% purity) was purified first by vacuum distillation at $50 \mathrm{mT}$ Torr for five times. The distilled sample, collected in a $17-\mathrm{mm}$ diameter Pyrex ampoule ( $2.5 \mathrm{~mm}$ in thickness), was further purified by three-zone refining for more than 60 passes. The purified sample was processed by directional solidification, and the dynamics of crystal growth was studied. No morphological breakdown was observed up to $7 \mu \mathrm{m} / \mathrm{s}$ of the solidification speed for a thermal gradient of $8-10 \mathrm{~K} / \mathrm{cm}$. To make the melting temperature dependent on the concentration, about $0.064 \mathrm{wt} \%$ of acetone was injected into the sample through a $5 \mu 1$ micro-syringe inserted into the bottom of the sample. The total sample length was about $20 \mathrm{~cm}$.

The Bridgman furnace consisted of two heating zones made of insulated copper blocks each with a nichrome wire inside as a heating element. In between, a transparent insulation zone made of Plexiglas was used. The hot- and cold-zone temperatures were controlled independently by two PID controllers and the temperatures were set at $80^{\circ} \mathrm{C}$ a

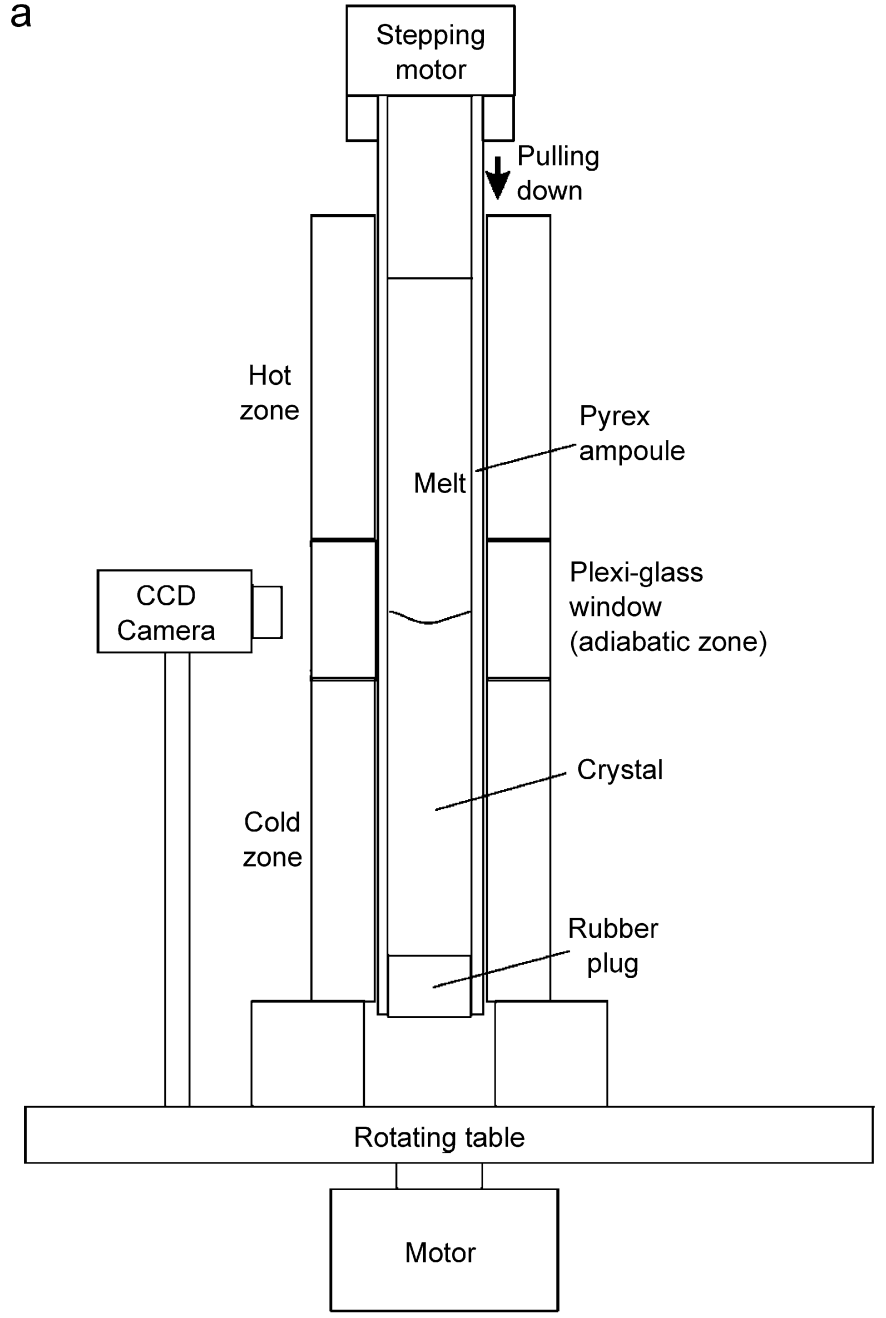

b

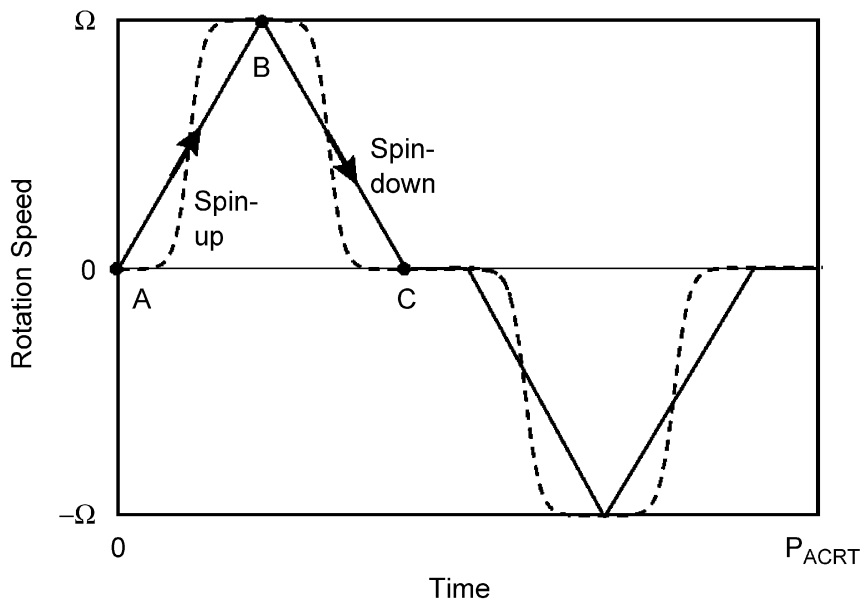

Fig. 1. (a) Schematic of the transparent vertical Bridgman system on a rotating table, (b) the ACRT rotation cycles used in this study; the time for spin-up and spin-down is $5 \mathrm{~s}$ and the resting time $2 \mathrm{~s} ; P_{\mathrm{ACRT}}=24 \mathrm{~s}$; the dashed line is used for the amplitude at 20 RPM only.

(hot zone) and $40{ }^{\circ} \mathrm{C}$ (cold zone), respectively. To translate the ampoule accurately, a microstepping motor was used to drive a screw slide; the translation rate was controlled at $1.6 \mu \mathrm{m} / \mathrm{s}$ in this study. During crystal growth, a digital 
video camera recorded the evolution of the interface morphology with a back lighting to enhance the contrast of the image. Because the camera was rotating with the sample, the images could be captured more clearly. ACRT was implemented through the rotating table controlled by a programmable motor controller. The rotation cycle pattern used in this study is shown by the solid line in Fig. 1b. A similar pattern was used in the previous simulation for VB growth of ZnCdTe [8-11]. It should be pointed out that the cycle pattern shown by the solid line in Fig. $1 \mathrm{~b}$ might not be exactly implemented in our system because of the inertial effect from the heavy aluminum table. We had further measured the table rotation speed. For 40 and $60 \mathrm{RPM}$, the rotation patterns were approximated reasonably by the triangular cycle pattern. However, for 20 RPM, the spin-up or down curve showed an $\mathrm{S}$ shape, as shown by the dashed line in Fig. 1b, rather than a straight line. As will be discussed shortly, this significantly affected the results.

To better understand the observed morphological evolution, an axisymmetric numerical model accounting for melt convection, heat and mass transfer, and the moving interface [13] was used to simulate the crystal growth process. The governing equations and boundary conditions are discretized by a finite volume method, and the resultant differential/algebraic equations are solved by DASPK solver with adaptive stepsize control. The total number of unknowns after the finite volume approximation is 34,052 , and all the calculations were performed in a personal computer (P4-3 GHz CPU). The benchmark comparison for the VB growth of $\mathrm{ZnCdTe}$ was performed using this computer program [11], and excellent agreement was found with previous calculations [8].

The physical properties for SCN are listed in Table 1 [14]. Also, the dimensionless numbers that are involved in the simulation are also listed. In short, the Prandtl number $\operatorname{Pr}(23.2)$ is much greater than 1 indicating that the effect of convection on heat transfer is significant. Similarly, the Schmidt number $S c$ being more than 2036 also implies the importance of the convective mass transfer. Moreover, near the interface, the estimated $\left.G r_{T}\right|_{\triangle T_{r}} / R e^{2}$ or $G r_{\mathrm{S}} / R e^{2}$, which is a measure of the significance of the buoyant convection as compared with the forced convection [15], is much smaller than unity. Accordingly, near the interface, the forced convection due to accelerated crucible rotation is dominant. Nevertheless, the buoyant convection away from the interface is still significant [8-11].

\section{Results and discussion}

Without using ACRT, the visualized interface evolution is shown in the first column of Fig. 2. As shown, before solidification was started, the interface was flat because the thermal conductivities of the melt and the crystal are very close to each other. As the ampoule moved downward at $1.6 \mu \mathrm{m} / \mathrm{s}$, the solidification began and the interface became concave due to the release of heat fusion, as shown by the
Table 1

Physical properties [13] and some input parameters

SCN/acetone

Solid density $\left(\rho_{\mathrm{c}}\right)=1016 \mathrm{~kg} / \mathrm{m}^{3}$

Melt density $\left(\rho_{\mathrm{m}}\right)=982 \mathrm{~kg} / \mathrm{m}^{3}$

Melting point $\left(T_{\mathrm{m}}\right)=331.233 \mathrm{~K}$

Heat of fusion $(\Delta H)=48380 \mathrm{~J} / \mathrm{kg}$

Thermal conductivity of solid and melt $\left(k_{\mathrm{c}}=k_{\mathrm{m}}\right)=0.224 \mathrm{~W} / \mathrm{m}-\mathrm{K}$

Specific heat of solid and melt $\left(C_{\mathrm{p}_{\mathrm{c}}}=C_{\mathrm{p}_{\mathrm{m}}}\right)=2000 \mathrm{~J} / \mathrm{kg}-\mathrm{K}$

Thermal expansion coefficient $\left(b_{\mathrm{T}}\right)=1.07 \times 10^{-3} \mathrm{~K}^{-1}$

Solutal expansion coefficient $\left(b_{\mathrm{S}}\right)=1 \times 10^{-3}(\mathrm{wt} \% \text { acetone })^{-1}$

Acetone diffusivity in $\operatorname{SCN}(D)=1.3 \times 10^{-9} \mathrm{~m}^{2} / \mathrm{s}$

Viscosity $\left(\mu_{\mathrm{m}}\right)=2.6 \times 10^{-3} \mathrm{~kg} / \mathrm{s}-\mathrm{m}$

Segregation coefficient $(K)=0.1$

Ampoule

Density $\left(\rho_{\mathrm{a}}\right)=2200 \mathrm{~kg} / \mathrm{m}^{3}$

Thermal conductivity $\left(k_{\mathrm{a}}\right)=3.26 \times 10^{-2} \mathrm{~W} / \mathrm{m}-\mathrm{K}$

Specific heat $\left(C_{\mathrm{p}_{\mathrm{a}}}\right)=1880 \mathrm{~J} / \mathrm{kg}-\mathrm{K}$

\section{Other input parameters}

System length $(L)=0.15 \mathrm{~m}$

Crystal diameter $\left(R_{\mathrm{c}}\right)=8.5 \times 10^{-3} \mathrm{~m}$

Ampoule outside diameter $\left(R_{\mathrm{a}}\right)=1.1 \times 10^{-2} \mathrm{~m}$

Hot-zone temperature $\left(T_{\mathrm{H}}\right)=80^{\circ} \mathrm{C}$

Cold-zone temperature $\left(T_{\mathrm{C}}\right)=40^{\circ} \mathrm{C}$

Heat transfer coefficient $(h)=1600 \mathrm{~W} / \mathrm{m}^{2}-\mathrm{K}$

Ampoule moving speed $\left(U_{\mathrm{a}}\right)=-1.6 \times 10^{-6} \mathrm{~m} / \mathrm{s}$

Maximum rotation speed $(\Omega)=20-60 \mathrm{RPM}$

Dimensionless groups

Prandtl number $\operatorname{Pr}=\frac{C p_{m} \mu_{m}}{k_{m}}=23.21$

Schmidt number $S c=\frac{\mu_{m}}{\rho_{m} D}=2.04 \times 10^{3}$

Thermal Grashof number $G r_{\mathrm{T}}=\frac{g \beta_{T}\left(T_{H}-T_{C}\right) R_{c}^{3}}{\left(\mu_{m} / \rho_{m}\right)^{2}}=3.67 \times 10^{4}$

Based on radial temperature difference

$\left(\Delta T_{r} \approx G \times\left(\right.\right.$ interface concavity) $\left.=2^{\circ} C\right)$ :

$\left.G r_{T}\right|_{\triangle T_{r}}=G r_{T} \times\left(\triangle T_{r} / \triangle T\right)=4317.64$

Solutal Grashof number $G r_{S}=\frac{g \beta_{S} C_{0} R_{c}^{3}}{\left(\mu_{m} / \rho_{m}\right)^{2}}=54.9$

Taylor number $T a=\frac{4 \Omega^{2} R_{c}^{4}}{\left(\mu_{m} / \rho_{m}\right)^{2}}=1.18 \times 10^{5}$ (for $\left.60 \mathrm{RPM}\right)$

Reynolds number $R e=\frac{\Omega R_{c}^{2}}{\left(\mu_{m} / \rho_{m}\right)}=171.45$ (for $60 \mathrm{RPM}$ )

photograph at $20 \mathrm{~min}$. At $40 \mathrm{~min}$, a clear depression was formed at the interface center due to acetone accumulation that lowered the solidification temperature. A pit was also found at the center of the interface similar to that reported previously [16]; however, it was not clearly seen here because of the improper lighting condition. Meanwhile, the morphological breakdown, as a result of constitutional supercooling, began at around $40 \mathrm{~min}$. The breakdown area spread out more from 60 to $80 \mathrm{~min}$. Then, the area size remained about the same till the end of the experiment at $120 \mathrm{~min}$.

With ACRT having the amplitude of 20 RPM, the interface concavity and the depression area slightly increased as shown by the photograph in the second column of Fig. 2 at $40 \mathrm{~min}$. Meanwhile, the morphological breakdown was more and clearer; the onset of the supercooling was found slightly earlier than the case of no rotation. At $60 \mathrm{~min}$, the breakdown area became much larger and then slightly increased until $80 \mathrm{~min}$, after which 


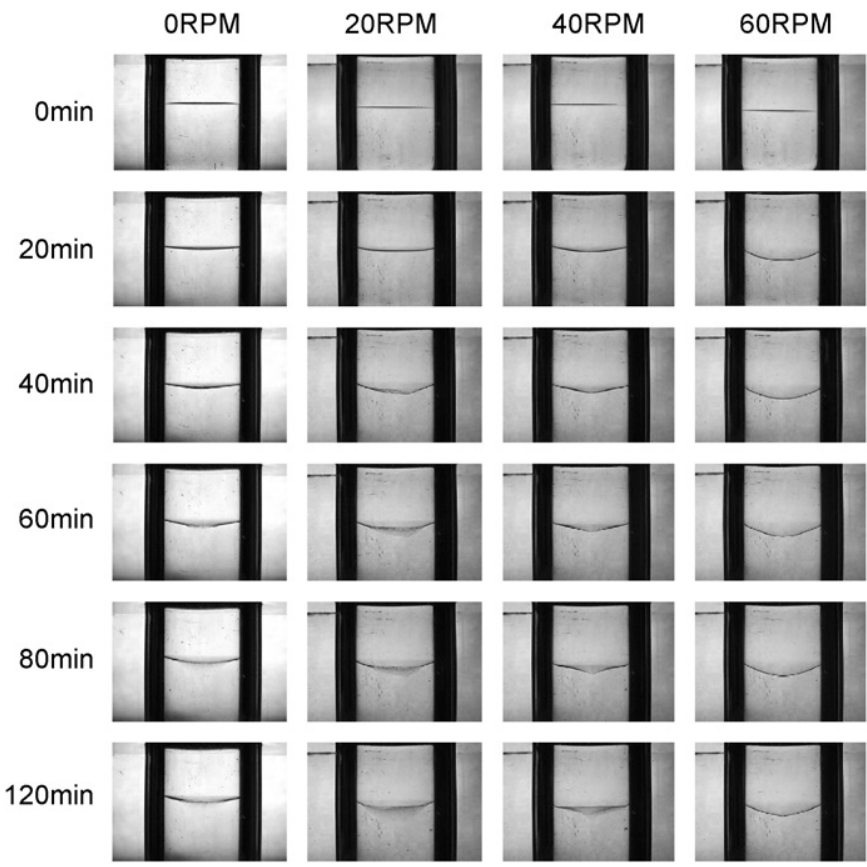

Fig. 2. Observed morphological evolutions during solidification of SCN containing $0.068 \mathrm{wt} \%$ acetone at different ACRT amplitudes.

the change was not significant till the end of the experiment. Increasing the rotation amplitude to 40 RPM, as shown by the third column, the interface concavity at $20 \mathrm{~min}$ increased further, but the interface became sharper near the center. The onset of the morphological breakdown was significantly delayed until almost $80 \mathrm{~min}$. Also, the breakdown occurred rather uniformly over the interface except the area near the ampoule wall. The triangular shape of the interface near the center of interface was quite different from the one at 20 RPM.

As the rotation amplitude was increased to $60 \mathrm{RPM}$, the interface concavity further increased and stabilized quickly in $40 \mathrm{~min}$, as shown by the last column in Fig. 2. The concavity remained about the same till the end of the experiment. Interestingly, no morphological breakdown was observed in $120 \mathrm{~min}$. Because SCN is an organic material having a Prandtl number $P r$ of 23.2, the effect of flow on the heat transfer and thus the interface shape is believed to be significant. In fact, in the previous simulations [10,11], it was illustrated that ACRT could increase the interface concavity for ZnCdTe. Nevertheless, the increase of the concavity was not as large as the one here.

The evolution of the interface morphology with the rotation amplitude shown in Fig. 2 cannot be explained easily without the assistance of computer simulation. Hence, computer simulation is also carried out for the experiments using the rotation pattern shown by the solid line in Fig. 1b. For the case of no rotation, the simulated results are shown in Fig. 3. As shown, at stationary, the interface is almost flat, which is consistent with the

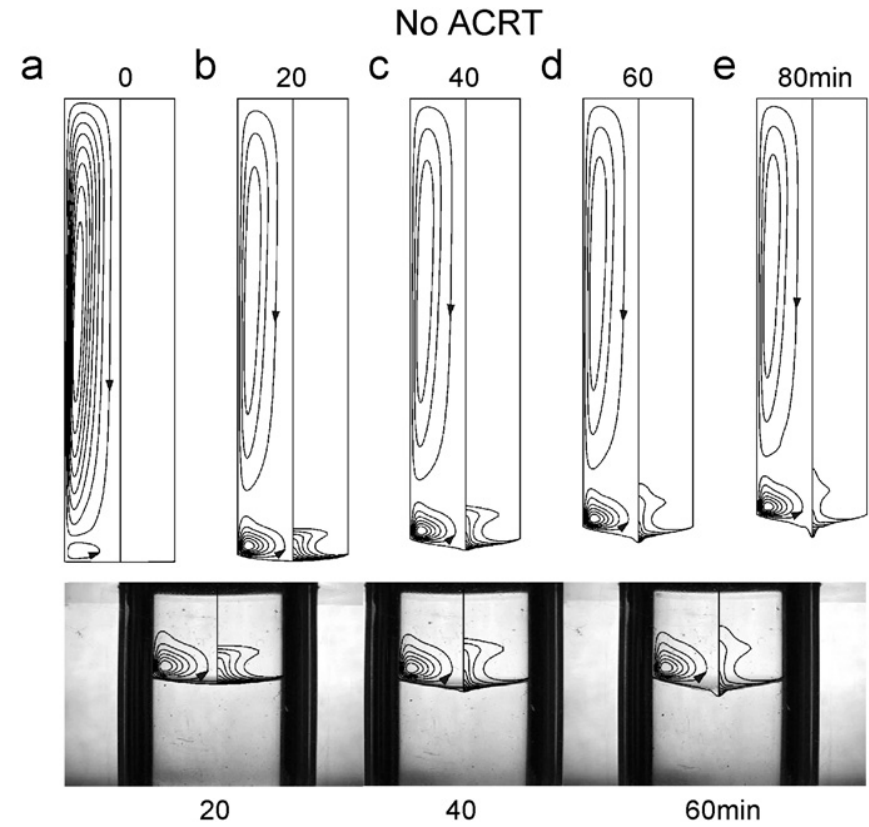

Fig. 3. Calculated flow (left) and acetone (right) fields for the growth without rotation: $\Psi_{\min }=-1.798 \times 10^{-4} \mathrm{~g} / \mathrm{s}, \quad \Psi_{\max }=3.234 \times 10^{-5} \mathrm{~g} / \mathrm{s}$, $C_{\min } / C_{0}=1, C_{\max } / C_{0}=1$ in (a), $\Psi_{\min }=-1.489 \times 10^{-4} \mathrm{~g} / \mathrm{s}, \quad \Psi_{\max }=$ $2.630 \times 10^{-4} \mathrm{~g} / \mathrm{s}, \quad C_{\min } / C_{0}=1, \quad C_{\max } / C_{0}=2.803 \quad$ in $\quad(\mathrm{b}), \quad \Psi_{\min }=$ $-1.489 \times 10^{-4} \mathrm{~g} / \mathrm{s}, \quad \Psi_{\max }=2.859 \times 10^{-4} \mathrm{~cm}^{3} / \mathrm{s}, C_{\min } / C_{0}=1, C_{\max } / C_{0}=$ 5.503 in (c), $\Psi_{\min }=-1.488 \times 10^{-4} \mathrm{~g} / \mathrm{s}, \Psi_{\max }=2.867 \times 10^{-4} \mathrm{~g} / \mathrm{s}, C_{\min } / C_{0}$ $=0.992, \quad C_{\max } / C_{0}=8.643$ in $(\mathrm{d}), \quad$ and $\quad \Psi_{\min }=-1.487 \times 10^{-4} \mathrm{~g} / \mathrm{s}$, $\Psi_{\max }=2.833 \times 10^{-4} \mathrm{~g} / \mathrm{s}, C_{\min } / C_{0}=0.975, C_{\max } / C_{0}=13.934$ in (e).

experimental observation. The flat interface at stationary is because the thermal conductivity of the melt is about the same as the solid. A very small convection cell, counterclockwise in direction, is induced near the interface. The main flow cell in the melt, clockwise in direction, is caused by the radial heating from the heater. As the solidification starts, the interface concavity increases as a result of the release of heat of fusion. Due to the larger radial thermal gradients induced near the interface, the flow near the interface becomes stronger causing acetone accumulation at the center of the interface, as that shown in Fig. 3b. As the solidification continues, as shown in Fig. 3c at 40 min, the acetone accumulates and a small depression forms at the center of the interface; eventually, the constitutional supercooling occurs before $40 \mathrm{~min}$ of solidification. The numerical breakdown is encountered after $60 \mathrm{~min}$ as the supercooling builds up to a certain degree. We also overlap the calculated interface shapes with the photographs in the bottom of Fig. 3 for comparison. As shown, the interface shapes before breakdown are in good agreement. Again, the pit was not clearly seen in the photographs; however, a clear pit was found during experiments as the one similar to that observed in Ref. [16].

With ACRT, we take the cases for 40 and 60 RPM for illustration first; the cycle pattern is shown by the solid line in Fig. 1b. As shown in Fig. 4 for 40 RPM, after ACRT starts, the upper flow cell is similar to the one without ACRT, but its intensity is much smaller than the flow cell 
40 RPM ACRT

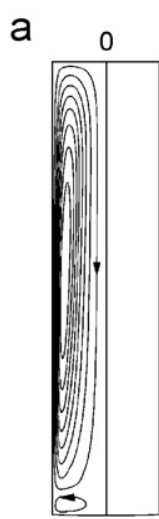

b

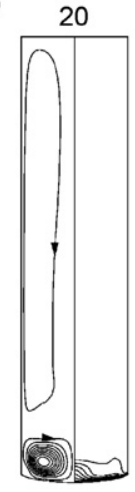

$\mathrm{C}$

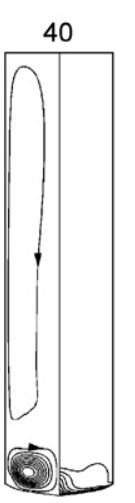

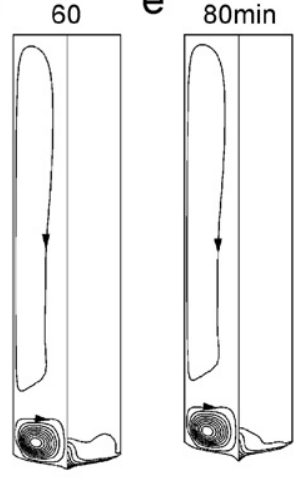

d 60 e

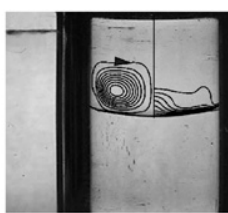

20

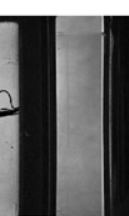

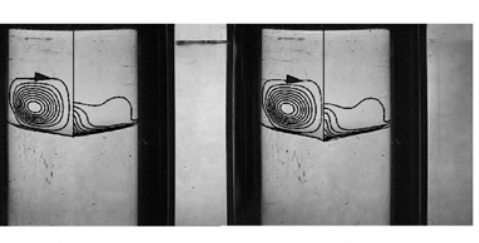

40

$60 \mathrm{~min}$
Fig. 4. Calculated flow (left) and acetone concentration (right) fields at $40 \mathrm{RPM} \quad$ ACRT: $\Psi_{\min }=-1.798 \times 10^{-4} \mathrm{~g} / \mathrm{s}, \quad \Psi_{\max }=3.234 \times 10^{-5} \mathrm{~g} / \mathrm{s}$, $C_{\min } / C_{0}=1, \quad C_{\max } / C_{0}=1$ in (a), $\Psi_{\min }=-1.844 \times 10^{-3} \mathrm{~g} / \mathrm{s}, \quad \Psi_{\max }=$ $1.670 \times 10^{-4} \mathrm{~g} / \mathrm{s}, \quad C_{\min } / C_{0}=0.999, \quad C_{\max } / C_{0}=2.013$ in $(\mathrm{b}), \quad \Psi_{\min }=$ $-1.838 \times 10^{-3} \mathrm{~g} / \mathrm{s}, \quad \Psi_{\max }=1.630 \times 10^{-4} \mathrm{~g} / \mathrm{s}, \quad C_{\min } / C_{0}=0.999, \quad C_{\max } /$ $C_{0}=4.004$ in (c), $\Psi_{\min }=-1.841 \times 10^{-3} \mathrm{~g} / \mathrm{s}, \quad \Psi_{\max }=1.683 \times 10^{-4} \mathrm{~g} / \mathrm{s}$, $C_{\min } / C_{0}=0.999, C_{\max } / C_{0}=6.093$ in (d), and $\Psi_{\min }=-1.483 \times 10^{-3} \mathrm{~g} / \mathrm{s}$, $\Psi_{\max }=1.711 \times 10^{-4} \mathrm{~cm}^{3} / \mathrm{s}, C_{\min } / C_{0}=0.999, C_{\max } / C_{0}=9.344$ in (e).

near the interface. The instantaneous flow structure near the interface at different growth stages is at the beginning of the spin-up stage (point A in Fig. 1b); the flow structure at the spin-down stage and the cycle-averaged flow will be discussed shortly. The lower flow cell is clockwise in direction, which is different from the previous one caused by the buoyancy force. As compared with the interface shapes shown in the third column in Fig. 3, the simulated interface shapes are consistent with the observed ones. For example, as the one at $60 \mathrm{~min}$, the interface shape becomes more curved near the ampoule wall, but shaper at the center. As shown by the comparison at the bottom in Fig. 4, this triangular shape is indeed consistent with the observed one.

At 60 RPM, as shown in Fig. 5, a significant change in the interface shape is found. The interfaces become quite concave as the ones observed in the experiments; as shown by the bottom plots in Fig. 5, the agreement of the calculated interface shapes with the observed ones is reasonably good. Because the lower flow cell is much stronger than the upper cell, at the same flow direction, the streamlines of the upper cell do not appear with the plotting levels used here. Some instantaneous flow patterns and acetone concentrations corresponding to the stages $\mathrm{A}$, B, and $\mathrm{C}$ indicated in Fig. $1 \mathrm{~b}$ are shown in Fig. 6. The spinup flows at 3600 and $3605 \mathrm{~s}$ near the interface are counterclockwise in direction. The spin-down flow at

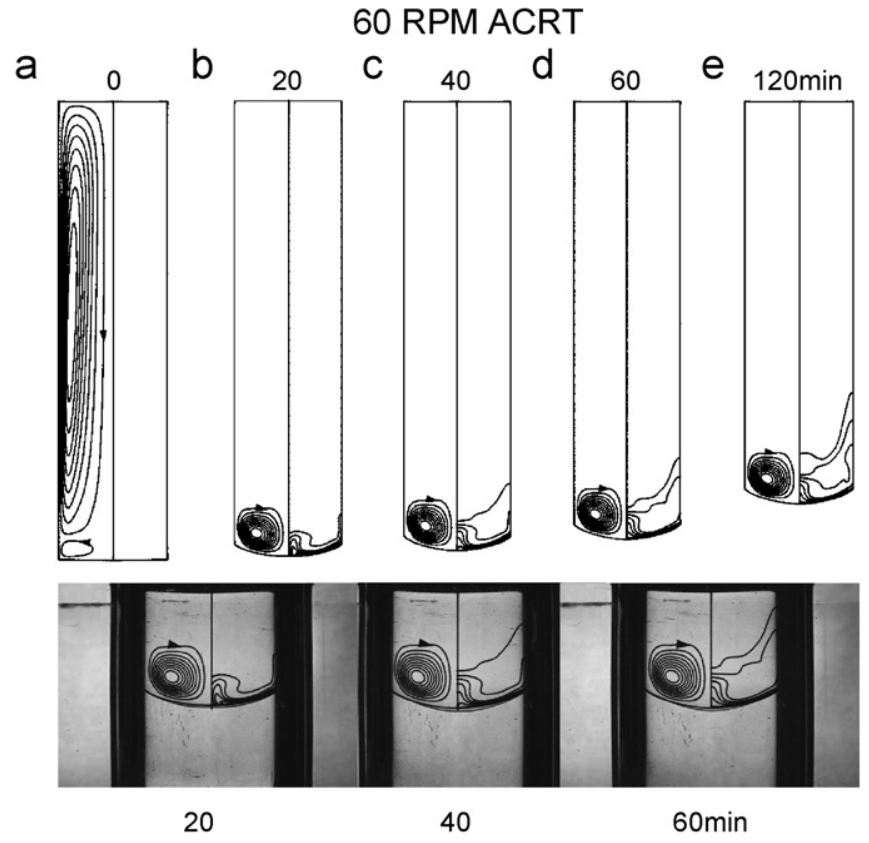

Fig. 5. Calculated flow (left) and acetone concentration (right) fields at $60 \mathrm{RPM}$ ACRT. $\Psi_{\min }=-1.798 \times 10^{-4} \mathrm{~g} / \mathrm{s}, \quad \Psi_{\max }=3.234 \times 10^{-5} \mathrm{~g} / \mathrm{s}$, $C_{\min } / C_{0}=1, C_{\max } / C_{0}=1$ in (a), $\Psi_{\min }=-3.830 \times 10^{-3} \mathrm{~g} / \mathrm{s}, \quad \Psi_{\max }=$ $1.678 \times 10^{-4} \mathrm{~g} / \mathrm{s}, \quad C_{\min } / C_{0}=0.988, \quad C_{\max } / C_{0}=1.630$ in (b), $\Psi_{\min }=$ $-3.819 \times 10^{-3} \mathrm{~g} / \mathrm{s}, \quad \Psi_{\max }=1.630 \times 10^{-4} \mathrm{~g} / \mathrm{s}, \quad C_{\min } / C_{0}=0.998, \quad C_{\max } /$ $C_{0}=2.171$ in (c), $\Psi_{\min }=-3.823 \times 10^{-3} \mathrm{~g} / \mathrm{s}, \quad \Psi_{\max }=1.724 \times 10^{-4} \mathrm{~g} / \mathrm{s}$, $C_{\min } / C_{0}=0.999, C_{\max } / C_{0}=2.531$ in (d), and $\Psi_{\min }=-3.086 \times 10^{-3} \mathrm{~g} / \mathrm{s}$, $\Psi_{\max }=1.645 \times 10^{-4} \mathrm{~cm}^{3} / \mathrm{s}, C_{\min } / C_{0}=0.999, C_{\max } / C_{0}=3.307$ in (e).

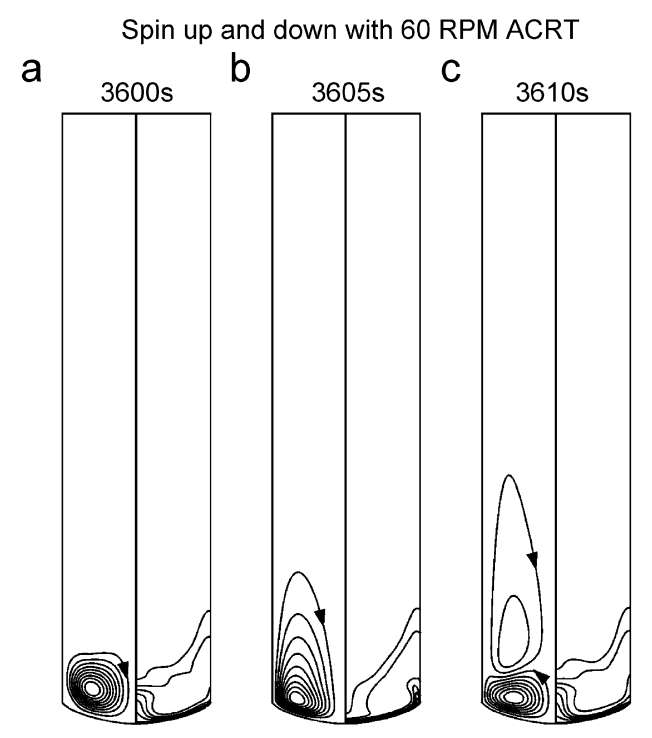

Fig. 6. Calculated flow (right) and acetone concentration (left) fields: (a) $3600 \mathrm{~s}$ ( $2 \mathrm{~s}$ after the spin-down cycle); (b) $3605 \mathrm{~s}$ (spin-up); (c) $3610 \mathrm{~s}$ (spindown). $\quad \Psi_{\min }=-1.659 \times 10^{-3} \mathrm{~g} / \mathrm{s}, \quad \Psi_{\max }=8.096 \times 10^{-3} \mathrm{~g} / \mathrm{s}, \quad C_{\min } /$ $C_{0}=0.999, \quad C_{\max } / C_{0}=2.531 \quad$ in $\quad(\mathrm{a}), \quad \Psi_{\min }=-1.687 \times 10^{-3} \mathrm{~g} / \mathrm{s}$, $\Psi_{\max }=8.244 \times 10^{-3} \mathrm{~g} / \mathrm{s}, C_{\min } / C_{0}=0.999, C_{\max } / C_{0}=2.441$ in (b), and $\Psi_{\min }=-1.696 \times 10^{-3} \mathrm{~g} / \mathrm{s}, \quad \Psi_{\max }=8.244 \times 10^{-3} \mathrm{~g} / \mathrm{s}, \quad C_{\min } / C_{0}=0.999$, $C_{\max } / C_{0}=2.366$ in (c).

$3605 \mathrm{~s}$ is at the opposite flow direction. The acetone fields are significantly affected by the instantaneous flow as well. Similarly, the isotherms, not shown here, near the interface 
a

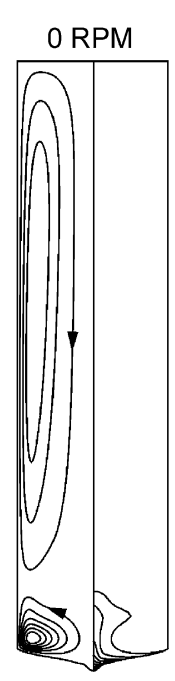

b

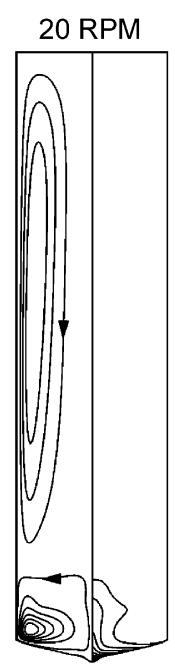

C

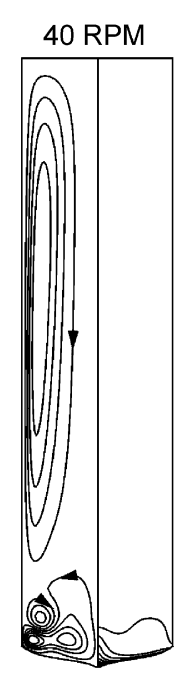

d

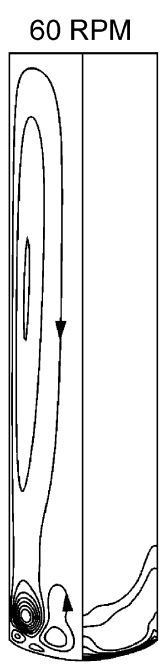

Fig. 7. Calculated averaged flow (left) and acetone consternation (right) fields at $1 \mathrm{~h}$ for ACRT with different rotation speeds: (a) 0 RPM; (b) 20 RPM; (c) 40 RPM; and (d) 60 RPM; the cycle pattern is shown by the solid line in Fig. $1 \mathrm{~b} . \Psi_{\min }=-1.489 \times 10^{-4} \mathrm{~g} / \mathrm{s}, \Psi_{\max }=2.857 \times 10^{-4} \mathrm{~g} / \mathrm{s}$, $C_{\min } / C_{0}=1, \quad C_{\max } / C_{0}=5.392 \quad$ in $\quad\left(\right.$ a),$\quad \Psi_{\min }=-1.488 \times 10^{-4} \mathrm{~g} / \mathrm{s}$, $\Psi_{\max }=2.291 \times 10^{-4} \mathrm{~g} / \mathrm{s}, \quad C_{\min } / C_{0}=0.999, \quad C_{\max } / C_{0}=5.088 \quad$ in (b), $\Psi_{\min }=-1.485 \times 10^{-4} \mathrm{~g} / \mathrm{s}, \quad \Psi_{\max }=1.545 \times 10^{-4} \mathrm{~g} / \mathrm{s}, \quad C_{\min } / C_{0}=0.999$, $C_{\max } / C_{0}=3.824$ in (c), and $\Psi_{\min }=-4.529 \times 10^{-4} \mathrm{~g} / \mathrm{s}, \quad \Psi_{\max }=$ $1.534 \times 10^{-4} \mathrm{~g} / \mathrm{s}, C_{\min } / C_{0}=0.998, C_{\max } / C_{0}=2.049$ in (d).

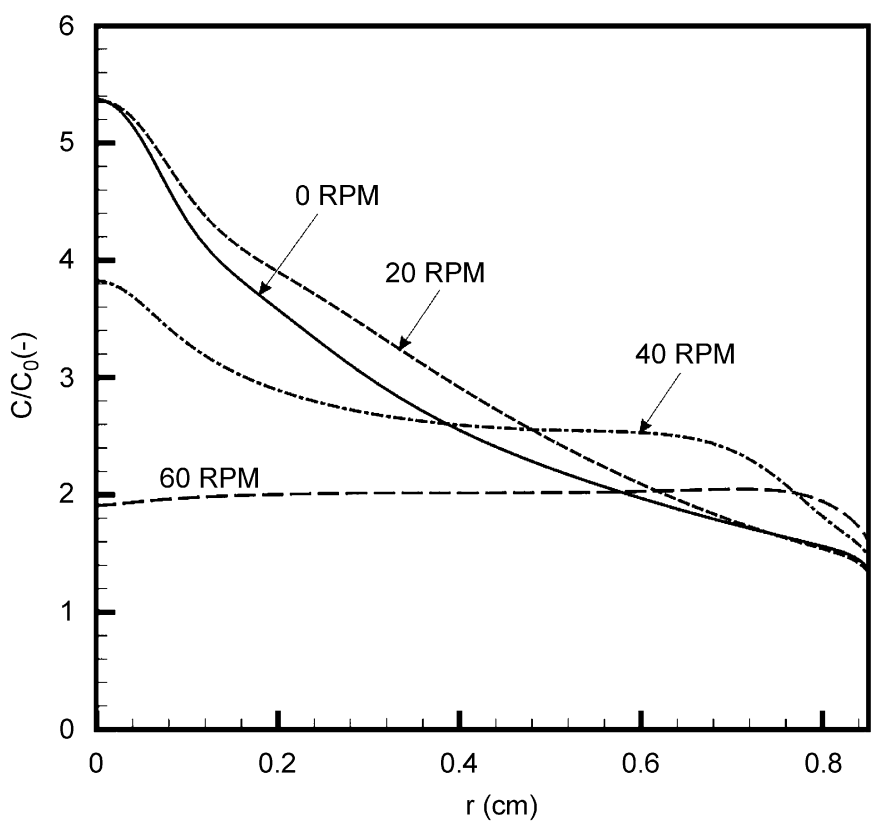

Fig. 8. Calculated averaged acetone radial concentration profiles at different ACRT amplitudes.

are found distorted toward the center of the interface. As a result, the interface becomes significantly concave at 60 RPM.

The averaged flow and acetone fields for different ACRT amplitudes at $60 \mathrm{~min}$ after solidification are shown in Fig. 7 , from which the acetone concentration profiles at the interface are plotted in Fig. 8. As shown in Fig. 7, it is clear that the original upper cell is not affected much by ACRT, except at $60 \mathrm{RPM}$. In other words, the solute mixing is confined in the space near the interface. However, at 60 RPM, the global mixing is slightly enhanced due to the connection of the lower and the upper flow cells. Therefore, the maximum acetone concentration is significantly lower for $60 \mathrm{RPM}$. On the other hand, the lower cell is affected significantly by ACRT and this significantly affects the radial acetone segregation. As shown in Fig. 8, in general, as the rotation amplitude increases, except 20 RPM, the acetone accumulation at the interface center decreases and the radial uniformity increases for the acetone concentration at the interface.

More importantly, as a results from the averaged flow structures and acetones fields, the calculated interface shapes shown in Fig. 7 are in reasonable agreement with the observed ones, except for 20 RPM. The more curved shape near the ampoule at 40 RPM is caused by the corner flow cell as a result of the spin-up effect. At 60 RPM, the lower center cell is much weaker, and the spin-up flow prevails leading to the significantly concave interface.

From Fig. 8, it is clear that ACRT improves radial acetone uniformity and lowers its concentration due to the improved mixing by ACRT. Particularly, at 60 RPM, the much slower acetone accumulation, as a result of a better global mixing, reduces supercooling and enhances the morphological stability. The more acetone accumulation at 20 RPM could also explain the slightly earlier morphological breakdown shown in Fig. 2.

To further examine the onset of the morphological instability, the superheating gradients $\Delta G$, as defined in Eq. (1), at the centerline are calculated. Without ACRT, the time history of $\Delta G$ is shown in Fig. 9a. As shown, before crystal growth, the acetone concentration is uniform and $\Delta G=G \sim 10 \mathrm{~K} / \mathrm{cm}$. Once the solidification starts, the acetone starts to accumulate in front of the interface (the segregation coefficient of acetone is 0.1 ), the superheating gradient decreases. When $\Delta G$ is less than zero, the constitutional supercooling occurs. Without ACRT, the supercooling occurs at $2300 \mathrm{~s}$ (slightly shorter than $40 \mathrm{~min}$ observed in the experiments). With $20 \mathrm{RPM}$ ACRT, as shown in Fig. $9 \mathrm{~b}, \Delta G$ oscillates significantly with the rotation cycles. The averaged $\Delta G$ reaches zero at about $2500 \mathrm{~s}$ (slightly longer than $40 \mathrm{~min}$ ). The early appearance of the morphological breakdown observed in the experiments, as the one shown in the second column in Fig. 2, could be the cause of the instantaneous supercooling due to flow oscillations. For 40 RPM ACRT, as shown in Fig. 9c, the oscillation amplitude of $\Delta G$ increases and the averaged supercooling appears after $50 \mathrm{~min}$. Interestingly, there is no averaged supercooling in $120 \mathrm{~min}$ for $60 \mathrm{RPM}$ ACRT, as shown in Fig. 9d. Nevertheless, the oscillation amplitude is more than three times of the initial thermal gradient. As compared with the observation in the experiments, it may be concluded that the averaged supercooling could be a reasonable criterion for the onset of the morphological breakdown. Indeed for $\mathrm{SCN}$, the interfacial energy contribution is small, so that its stability parameter [17] is 
a

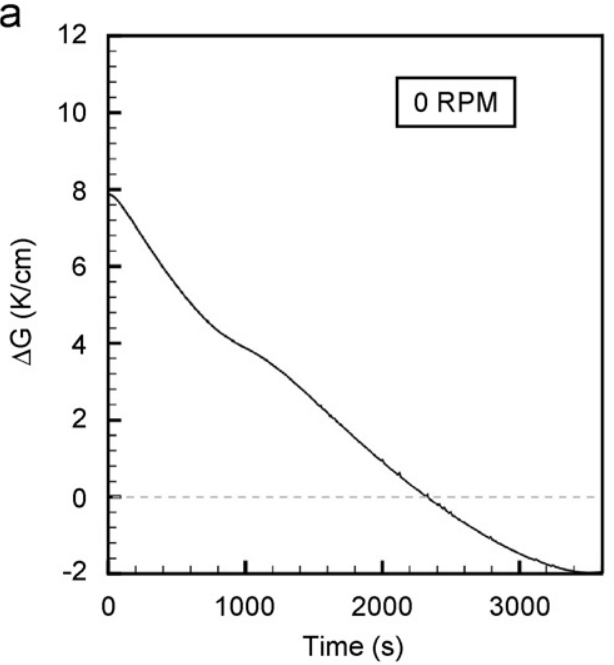

C

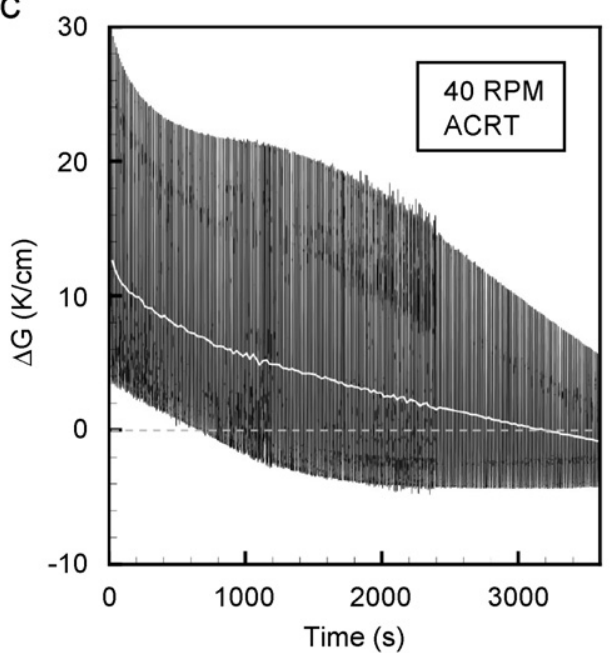

b

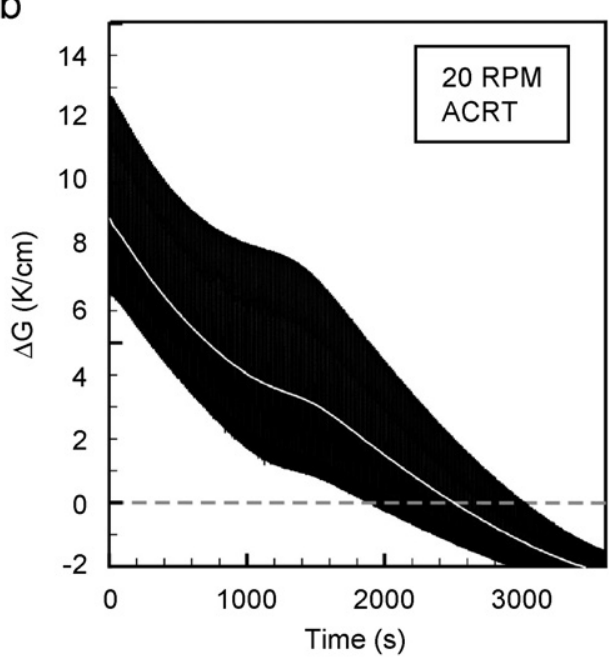

d

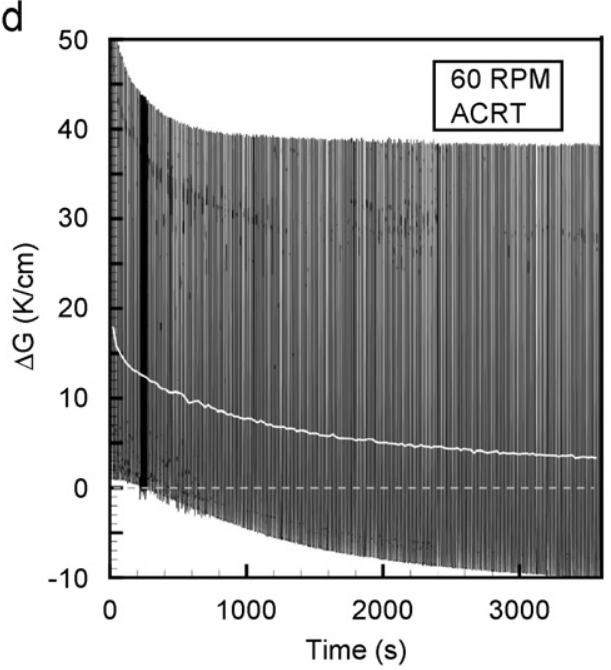

Fig. 9. Effect of ACRT amplitudes on the calculated superheating history at the center of the interface: (a) 0 RPM; (b) 20 RPM; (c) 40 RPM; and (d) 60 RPM; the cycle averaged values are indicated by the white lines from (b) to (c).

near unity and the supercooling is a good criterion for morphological breakdown.

So far, we have not discussed the poor prediction of the interface shape and morphological instability from simulation for 20 RPM. As mentioned in Section 2, due to the inertial effect of the rotating table, the rotation cycle pattern shown by the solid line in Fig. 1b could not be generated nicely. A more realistic representation of the rotation cycle might resemble the dashed line in Fig. 1b, the simulation may a better result. The simulated results based on the old and new rotation schedules are shown in Figs. $10 \mathrm{a}$ and $\mathrm{b}$, respectively. As shown, the new rotation schedule gives a wider interface shape and the acetone distribution near the interface, which is more consistent with experimental observation. The comparison of the simulated interface shapes using the new rotation cycle pattern with the observed ones, as shown in the bottom of Fig. $10 \mathrm{~b}$, is quite positive that the rotation cycle pattern is indeed important to the interface shape. Furthermore, the flow cell induced upon the lower cell also suppresses the acetone transport toward the bulk melt. Therefore, the confined space for acetone is further reduced causing the faster acetone accumulation there; one can see the higher $C_{\max } / C_{0}$ values in the acetone fields shown in Fig. $10 \mathrm{~b}$ as compared with that in Fig. 10a. This may further explain the earlier and wider breakdown area on the interface for 20 RPM. On the other hand, during experiments, we also observed that the interface shape often became asymmetric at low rotation amplitudes (less than 40 RPM). To further investigate this, we believe that a three-dimensional model is necessary, and we shall consider this in the near future.

\section{Conclusions}

The interface morphology during VB crystal growth of SCN containing $0.064 \mathrm{wt} \%$ acetone on a rotating stable was visualized for different ACRT amplitudes. Without rotation, the buoyancy convection led to a local acetone accumulation and accelerated constitutional supercooling at the center of the interface. Such a local accumulation 
a Old rotation cycle pattern

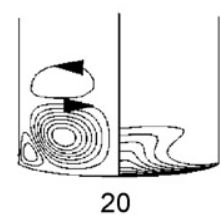

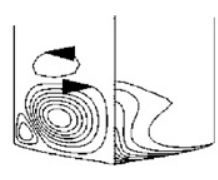

40

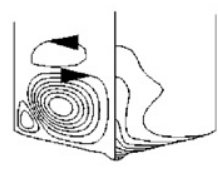

$60 \mathrm{~min}$ b

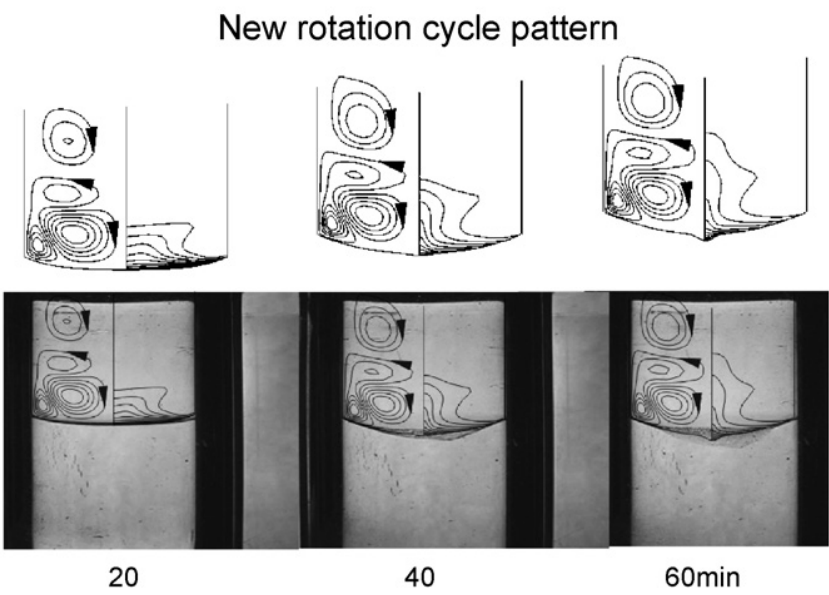

Fig. 10. Calculated flow and acetone fields for 20 RPM ACRT: (a) old rotation cycle pattern (the solid line in Fig. 1b) and (b) new rotation cycle pattern (the dashed line in Fig. 1b). The comparison of the interface shapes based on the new cycle with the observed ones is shown at the bottom of the figure. $\Psi_{\min }=-2.131 \times 10^{-4} \mathrm{~g} / \mathrm{s}, \Psi_{\max }=1.780 \times 10^{-4} \mathrm{~g} / \mathrm{s}$, $C_{\min } / C_{0}=0.999, \quad C_{\max } / C_{0}=2.325 ; \quad \Psi_{\min }=-1.876 \times 10^{-4} \mathrm{~g} / \mathrm{s}, \quad \Psi_{\max }=$ $1.973 \times 10^{-4} \mathrm{~g} / \mathrm{s}, \quad C_{\min } / C_{0}=0.999, \quad C_{\max } / C_{0}=4.844 ; \quad \Psi_{\min }=$ $-1.873 \times 10^{-4} \mathrm{~cm}^{3} / \mathrm{s}, \quad \Psi_{\max }=1.968 \times 10^{-4} \mathrm{~g} / \mathrm{s}, \quad C_{\min } / C_{0}=0.999, \quad C_{\max } /$ $C_{0}=7.410$ from the left to the right, respectively, in (a). $\Psi_{\min }=-4.365 \times 10^{-4} \mathrm{~g} / \mathrm{s}, \quad \Psi_{\max }=1.429 \times 10^{-4} \mathrm{~g} / \mathrm{s}, \quad C_{\min } / C_{0}=0.999$, $C_{\max } / C_{0}=2.552 ; \Psi_{\min }=-4.220 \times 10^{-4} \mathrm{~g} / \mathrm{s}, \quad \Psi_{\max }=1.590 \times 10^{-4} \mathrm{~cm}^{3} / \mathrm{s}$, $C_{\min } / C_{0}=0.999, \quad C_{\max } / C_{0}=5.365 ; \quad \Psi_{\min }=-4.215 \times 10^{-4} \mathrm{~g} / \mathrm{s}$, $\Psi_{\max }=1.583 \times 10^{-4} \mathrm{~g} / \mathrm{s}, C_{\min } / C_{0}=0.994, C_{\max } / C_{0}=8.371$ from the left to the right, respectively, in (b).

was reduced by increasing the rotation amplitude. Before a good mixing of acetone at around 60 RPM was achieved, the dramatic change of the interface morphologies and the breakdown patterns indicated a flow transition from a buoyant to spin-up/spin-down convections. Computer simulation was also carried out to support this argument, and a reasonable agreement was obtained. Meanwhile, the calculated interface shapes and the onset of the morphological breakdown are well predicted by simulation. The inconsistency between the simulated and observed interface shapes at 20 RPM is caused by the different rotation cycle patterns.

\section{Acknowledgments}

This work was sponsored by the National Science Council (NSC) of Taiwan and the Orchid program between NSC and France Science Foundation.

\section{References}

[1] H.J. Scheel, E.O. Schulz-Dubois, J. Crystal Growth 8 (1971) 304.

[2] P. Capper, J.C. Brice, C.L. Jones, W.G. Coates, J.J.G. Gosney, C.K. Ard, I. Kenworthy, J. Crystal Growth 89 (1988) 171.

[3] P. Capper, J.E. Harris, E. O'Keefe, C.L. Jones, C.K. Ard, P. Mackett, D. Dutton, Mater. Sci. Eng. B 16 (1993) 29.

[4] W.G. Coates, P. Capper, C.L. Jones, J.J.G. Gosney, C.K. Ard, I. Kenworthy, A. Clark, J. Crystal Growth 94 (1989) 959.

[5] P. Capper, J.J. Gosney, UK Patent 2098879A, 1982.

[6] J.C. Brice, Crystal Growth Processes, Wiley, New York, 1986.

[7] A. Yeckel, J.J. Derby, J. Crystal Growth 209 (2000) 734.

[8] A. Yeckel, J.J. Derby, J. Crystal Growth 233 (2001) 599.

[9] X. Liu, W. Jie, Y. Zhou, J. Crystal Growth 219 (2000) 22.

[10] X. Liu, W. Jie, Y. Zhou, J. Crystal Growth 209 (2000) 751.

[11] C.W. Lan, J. Crystal Growth 274 (2005) 379.

[12] W.A. Tiller, K.A. Jackson, J.W. Rutter, B. Chalmers, Acta Metall. 1 (1953) 428.

[13] C.W. Lan, M.C. Liang, J. Crystal Growth 186 (1998) 187.

[14] R.J. Schaefer, S.R. Coriell, Metall. Trans. A 15 (1984) 2109.

[15] U. Krzyminski, A.G. Ostrogorsky, J. Crystal Growth 174 (1997) 19.

[16] W.C. Yu, W.T. Hsu, B. Roux, T.P. Lyubimova, C.W. Lan, J. Crystal Growth 271 (2004) 474.

[17] R.F. Sekerka, in: P. Hartman (Ed.), Crystal Growth, North-Holland, Amsterdam, 1973, p. 403 